\title{
Magnetic Field Induced Transition in Vanadium Spinels
}

\author{
E. D. Mun, ${ }^{1}$ Gia-Wei Chern, ${ }^{2}$ V. Pardo,${ }^{3}$ F. Rivadulla, ${ }^{4}$ R. Sinclair,${ }^{5}$ H. D. Zhou, ${ }^{5}$ V. S. Zapf, ${ }^{1}$ and C. D. Batista ${ }^{2}$ \\ ${ }^{1}$ NHMFL Materials Physics and Applications, T-4 and CNLS, Los Alamos Laboratory (LANL), Los Alamos, New Mexico 87545, USA \\ ${ }^{2}$ Theoretical Division, T-4 and CNLS, Los Alamos Laboratory, Los Alamos, New Mexico 87545, USA \\ ${ }^{3}$ Departamento de Fisica Aplicada, Universidad de Santiago de Compostela, 15782 Santiago de Compostela, Spain \\ ${ }^{4}$ CIQUS, Universidad de Santiago de Compostela, 15782 Santiago de Compostela, Spain \\ ${ }^{5}$ Department of Physics and Astronomy, University of Tennessee, Knoxville, Tennessee 37996-1200, USA \\ (Received 16 July 2013; revised manuscript received 28 October 2013; published 10 January 2014)
}

\begin{abstract}
We study vanadium spinels $A \mathrm{~V}_{2} \mathrm{O}_{4}(A=\mathrm{Cd}, \mathrm{Mg})$ in pulsed magnetic fields up to $65 \mathrm{~T}$. A jump in magnetization at $\mu_{0} H \approx 40 \mathrm{~T}$ is observed in the single-crystal $\mathrm{MgV}_{2} \mathrm{O}_{4}$, indicating a field induced quantum phase transition between two distinct magnetic orders. In the multiferroic $\mathrm{CdV}_{2} \mathrm{O}_{4}$, the field induced transition is accompanied by a suppression of the electric polarization. By modeling the magnetic properties in the presence of strong spin-orbit coupling characteristic of vanadium spinels, we show that both features of the field induced transition can be successfully explained by including the effects of the local trigonal crystal field.
\end{abstract}

DOI: 10.1103/PhysRevLett.112.017207

PACS numbers: 75.10.Jm, 71.70.Gm, 75.50.Ee

The interplay between spin and orbital degrees of freedom of highly frustrated magnets becomes particularly relevant when the ground state manifold of the dominant interaction is massively degenerate [1]. This is the ideal scenario for finding rich phase diagrams because small interactions become the primary selection mechanism of spin-orbital ordering. A high susceptibility to small interactions opens the possibility of inducing phase transitions with moderate external pressure or magnetic fields. The vanadium spinels $A \mathrm{~V}_{2} \mathrm{O}_{4}(A=\mathrm{Cd}, \mathrm{Zn}$, and $\mathrm{Mg})$ are archetypical realizations of highly frustrated spin-orbital systems [2-12]. The magnetic $\mathrm{V}^{3+}$ ions reside on a frustrated pyrochlore lattice and contain two $d$ electrons in the three $t_{2 g}$ orbitals. These materials exhibit a cubic-to-tetragonal transition at a temperature $T=T_{S}$ and the onset of a $\mathbf{Q}=2 \pi(0,0,1)$ antiferromagnetic (AFM) ordering below $T_{N}<T_{S}$ [see Fig. 1(a)] [3-6]. The pyrochlore lattice can be viewed as a collection of cross-linking chains running along the $\langle 110\rangle$ directions. Below $T_{N}$, the $x y(z \| c$ axis) chains exhibit the usual Néel ordering, while chains oriented along the $x z$ and $y z$ directions exhibit an $\uparrow \uparrow \downarrow \downarrow$ superstructure [2-4]. This ordering induces an electric polarization $\mathbf{P} \simeq 5 \mu \mathrm{C} / \mathrm{m}^{2} \hat{\mathbf{z}}$ in $\mathrm{CdV}_{2} \mathrm{O}_{4}$ that arises from different oxygen displacements along $x z$ and $y z$ bonds [11], giving opposite contributions to $\mathbf{P}$ depending on whether the bond is ferromagnetic (FM) or AFM. Because the magnitude of the displacements is different for FM and AFM bonds, the $\uparrow \uparrow \downarrow \downarrow$ structure induces a net $\mathbf{P} \| \hat{\mathbf{z}}$.

The ground state manifold of these spinels is extensively degenerate if only nearest-neighbor (NN) exchange is included. The $\mathbf{Q}=2 \pi(0,0,1)$ ordering must then be selected by residual interactions which arise as a sequence of two selection mechanisms. The tetragonal distortion reduces the frustration by increasing the exchange along the $x y$ chains and inducing AFM spin correlations [4]. This distortion is accompanied by uniform occupation of the $x y$ orbitals and the onset of a magnetic easy $z$ axis. However, the exchange between crossing $x y$ chains remains frustrated. In the Mott limit relevant for $\mathrm{CdV}_{2} \mathrm{O}_{4}$, the $\uparrow \uparrow \downarrow \downarrow$ ordering is stabilized by a weak third NN AFM exchange [7]. For spinels near the Mott transition, like $\mathrm{ZnV}_{2} \mathrm{O}_{4}[13,14]$, the $\uparrow \uparrow \downarrow \downarrow$ superstructure could originate from Fermi point nesting of the quasi-1D $y z$ and $x z$ chains [15].

The weak nature of the interactions that select the magnetic ordering in highly frustrated magnets suggests that moderate fields could induce a different spin ordering. Indeed, multiple magnetic field induced transitions have been reported in pyrochlore antiferromagnets and spin ice $R_{2} \mathrm{Ti}_{2} \mathrm{O}_{7}$ [16-20]. While most transitions result from the competition between various spin-spin interactions and the Zeeman coupling, the presence of orbital degrees of freedom makes vanadium spinels ideal candidates for studying similar phenomena in frustrated spin-orbital systems. By including the subtle interplay between spin-orbit coupling and lattice distortion, our model predicts a new high-field magnetic order induced by a local trigonal distortion. Notably, although trigonal distortion in vanadium spinels has been discussed before [5,10], particularly in the ferrimagnetic spinel $\mathrm{MnV}_{2} \mathrm{O}_{4}$ [21-23], it has largely been neglected in the modeling of antiferromagnetic vanadium spinels.

Here we show experimental evidence of a quantum phase transition induced by relatively small fields in $\mathrm{MgV}_{2} \mathrm{O}_{4}$ and $\mathrm{CdV}_{2} \mathrm{O}_{4}$ spinels, in spite of their large Curie-Weiss temperature $\left|\Theta_{C W}\right| \simeq 300 \mathrm{~K}$ [6]. For a single crystal of $\mathrm{MgV}_{2} \mathrm{O}_{4}$, we observe a clear jump in the 


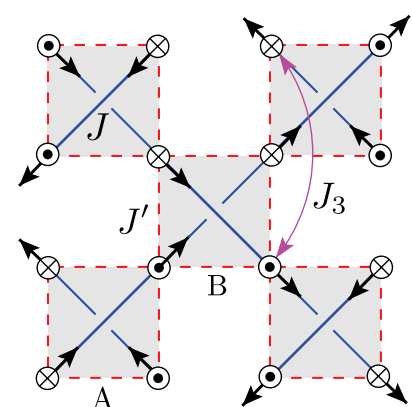

(a)

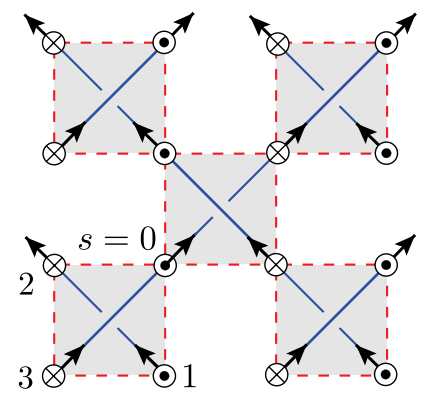

(b)
FIG. 1 (color online). Three-dimensional orderings for $A \mathrm{~V}_{2} \mathrm{O}_{4}$ viewed from [001] at $H=0$ (a) and predicted to occur at high fields (b). $\odot$ and $\otimes$ denote the $+z$ and $-z$ components of the moments. The arrows indicate the transverse spin components induced by the trigonal distortion. $J$ (solid blue), $J^{\prime}$ (dashed red), and $J_{3}$ are NN, next NN, and third NN exchange constants. $\mathrm{A}$ and $\mathrm{B}$ denote tetrahedra with opposite orientations.

magnetization at $\mu_{0} H \simeq 40 \mathrm{~T}$, while the multiferroic ordering of powder $\mathrm{CdV}_{2} \mathrm{O}_{4}$ is suppressed for $\mu_{0} H \gtrsim 30 \mathrm{~T}$. We explain this transition with a new selection mechanism based on a trigonal distortion, which is intrinsic to spinel structures and rotates the local easy axis toward the $\langle 111\rangle$ direction of each $\mathrm{V}^{3+}$. This rotation induces a weak FM component perpendicular to the Néel order parameter of each $x y$ chain (see Fig. 1). While the FM components of different chains cancel out for the low-field $\mathbf{Q}=(001)$ structure of Fig. 1(a), the $\mathbf{Q}=0$ spin ordering shown in Fig. 1(b) acquires a net transverse FM component, which makes it energetically favorable for large enough $H$. This new structure is not ferroelectric, which is in agreement with our experiments.

Reference [24] shows how the electronic structure of $A M_{2} X_{4}$ spinels containing $t_{2 g}$ electrons is affected by a varying trigonal field. One component arises from the trigonal distortion of the oxygen octahedron, as shown in Fig. 2. An opposing effect is caused by the surrounding trigonal antiprism of $t_{2 g}$-based metals ( $\mathrm{V}$ ions). Both contributions eventually cancel when moving from $\mathrm{ZnCr}_{2} \mathrm{O}_{4}$ to $\mathrm{HgCr}_{2} \mathrm{O}_{4}$ because the oxygen trigonal field increases while the $\mathrm{Cr}$ trigonal field decreases. For $\mathrm{CdV}_{2} \mathrm{O}_{4}$ and the structure proposed in Ref. [25], a very small tetragonal field is expected below $T_{S}$ that is accompanied by a larger trigonal distortion. The structure proposed in Ref. [11] (and also in Ref. [13] for the related compound $\mathrm{ZnV}_{2} \mathrm{O}_{4}$ ) explains the origin of the additional tetragonal term that further stabilizes the $d_{x y}$ orbital. From $a b$ initio calculations, we get $\Delta=$ $350 \mathrm{meV}$ and $\delta=250 \mathrm{meV}$ for the $t_{2 g}$ splittings induced by the tetragonal and trigonal distortions, respectively, [13].

We first present $P(H)$ and $M(H)$ data for $\mathrm{CdV}_{2} \mathrm{O}_{4}$ and show that the multiferroic $\uparrow \uparrow \downarrow \downarrow$ ordering of $\mathrm{CdV}_{2} \mathrm{O}_{4}$ is suppressed for $\mu_{0} H>30 \mathrm{~T}$. Similar features in $M(H)$ are observed in single-crystalline $\mathrm{MgV}_{2} \mathrm{O}_{4}$, which is too conductive to measure $P(H)$. Polycrystalline samples of
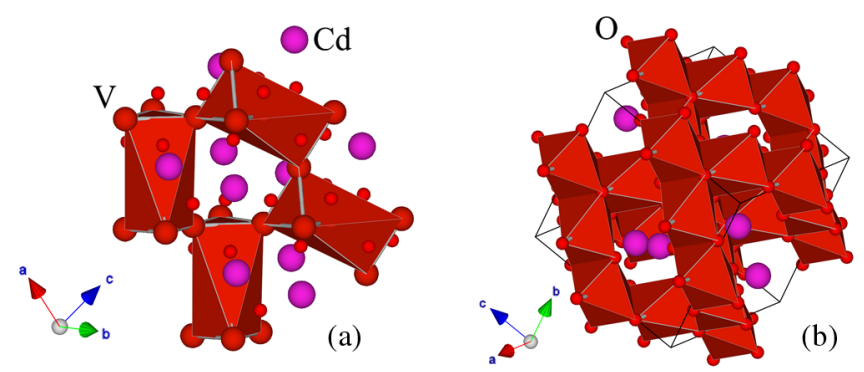

FIG. 2 (color online). (a) Trigonal antiprism environment of the neighboring $\mathrm{V}$ atoms. (b) Octahedra of $\mathrm{O}$ around the $\mathrm{V}$ atoms. (Pink circles with no bonds drawn are $\mathrm{Cd}^{2+}$ ions.) The two fields act in opposite directions, but they do not cancel out.

$\mathrm{CdV}_{2} \mathrm{O}_{4}$ were prepared by solid-state reaction, and the single crystals of $\mathrm{MgV}_{2} \mathrm{O}_{4}$ by a traveling floating zone method, as described in the Supplemental Material [26]. Low-field $M(H)$ and $P(H)$ measurements are consistent with previous publications $[11,27]$.

The high-field data are plotted in Fig. 3, and more details are described in the Supplemental Material [26]. $\mathrm{For}_{\mathrm{CdV}} \mathrm{O}_{4}$, $M(H)$ increases linearly until a field between 30 and $40 \mathrm{~T}$ and then shows an upturn for $T<T_{S}$ [see Fig. 3(a)]. The $M(H)$ curve of $\mathrm{CdV}_{2} \mathrm{O}_{4}$ reaches $0.4 \mu_{B} / \mathrm{V}^{3+}$ at $\mu_{0} H=65 \mathrm{~T}$, which is far below the saturated value for $\mathrm{V}^{3+} \mathbf{S}=1\left(2 \mu_{B} / \mathrm{V}^{3+}\right)$. By linear extrapolation, the saturation value would be reached for $\mu_{0} H>200 \mathrm{~T}$, consistent with $\left|\Theta_{C W}\right| \simeq 300 \mathrm{~K}$.
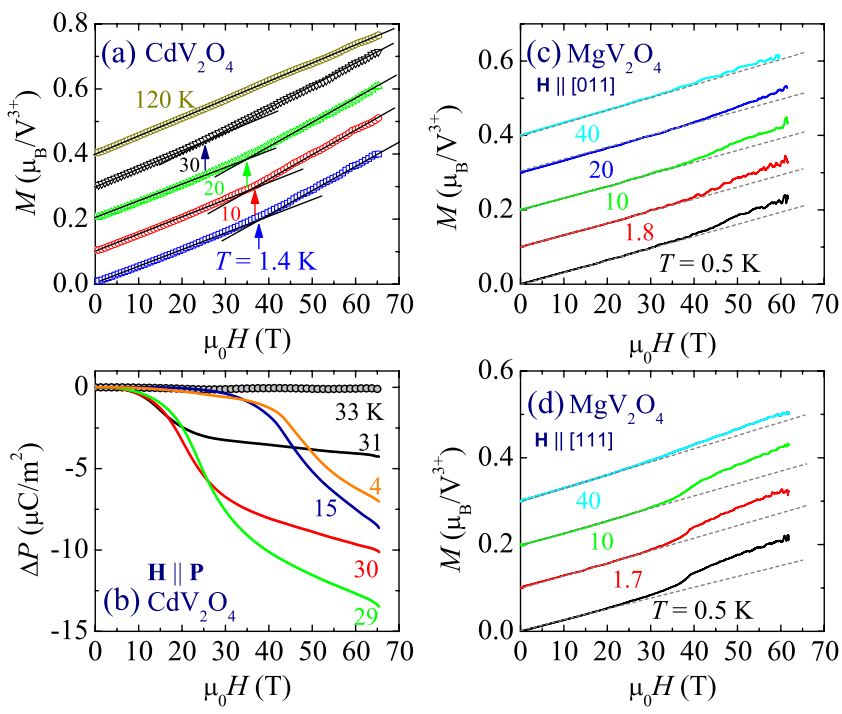

FIG. 3 (color online). (a) Magnetization isotherms, $M(H)$, of $\mathrm{CdV}_{2} \mathrm{O}_{4}$ at selected temperatures. Vertical arrows represent the critical field determined by drawing two straight lines. (b) $\Delta P(H)$ at selected temperatures determined by integrating the $d \Delta P / d t$ as a function of time. The sample was poled by applying external voltage across the contacts: $E_{P}=670 \mathrm{kV} / \mathrm{m}$. (c),(d) $M(H)$ of $\mathrm{MgV}_{2} \mathrm{O}_{4}$ at selected temperatures for orientations (1) and (2) described in the Supplemental Material [26]. For clarity, the $M(H)$ curves are successively shifted upward by $0.1 \mu_{B} / \mathrm{V}^{3+}$. Dashed lines are guides to the eye. 
Figure 3(b) shows $\Delta P(H)$ of $\mathrm{CdV}_{2} \mathrm{O}_{4}$ for $\mathbf{H} \| \mathbf{P}$ (similar data for $\mathbf{H} \perp \mathbf{P}$ are shown in the Supplemental Material [26]). $P(H)$ remains constant up to a field between 20 and $40 \mathrm{~T}$, depending on the temperature and then begins to decrease. No change in $P$ with $H$ is observed for $T>T_{N}=32.5 \mathrm{~K}$ (see $33 \mathrm{~K}$ curve). Just below $T_{N}(T=29 \mathrm{~K}), P(65 \mathrm{~T})-P(0)$ is close to the value of $\Delta P(T)$ across temperature-induced phase transition at $T_{N}$ [11], indicating that the $65 \mathrm{~T}$ field mostly suppresses ferroelectricity near $T_{N}$. However, $P(65 \mathrm{~T})-P(0)$ shrinks for $T \leq 29 \mathrm{~K}$, indicating that a finite $P$ remains for $H>65 \mathrm{~T}$. The residual ferroelectric moment at low $T$ here might be attributed to the polycrystalline nature of the sample. Grains with their $c$ axis roughly parallel to the field direction remain in the low-field state and, therefore, have a finite $P$.

Finally, Figs. 3(c) and 3(d) show the $M(H)$ curves of $\mathrm{MgV}_{2} \mathrm{O}_{4}$ for $\mathbf{H} \|[011]$ and $\mathbf{H} \|[111]$, respectively. $\mathrm{MgV}_{2} \mathrm{O}_{4}$ is too electrically conductive to measure electric polarization. Like for $\mathrm{CdV}_{2} \mathrm{O}_{4}, M(H)$ is linear up to a field between 30 and $40 \mathrm{~T}$ and then shows an upturn or a jump. In the Supplemental Material [26], we describe a magnetic hysteresis observed for $\mathbf{H} \|[111]$.

Figure 4 shows the $H-T$ phase diagram of $\mathrm{CdV}_{2} \mathrm{O}_{4}$ and $\mathrm{MgV}_{2} \mathrm{O}_{4}$ that results from the measured $M(H)$ and $\Delta P(H)$ curves. Because $\mathrm{CdV}_{2} \mathrm{O}_{4}$ is polycrystalline, we find it likely that the observed behaviors of $P(H)$ and $M(H)$ are caused by a single field induced transition that is broadened by anisotropy; i.e., the beginning of the upturn in $M(H)$ and downturn in $P(H)$ correspond to the transition field for $\mathbf{H}$ parallel to the easy axis, while the transition

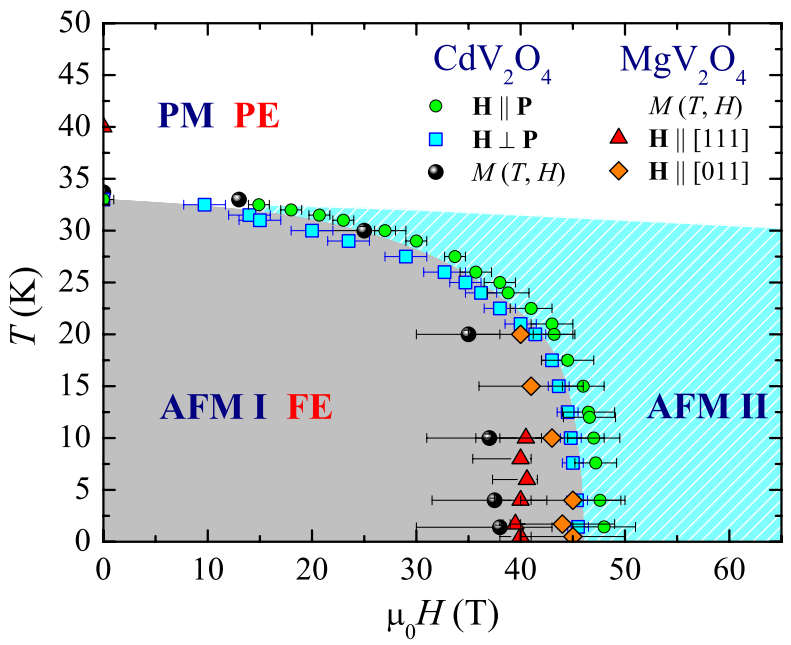

FIG. 4 (color online). $H$-T phase diagrams of $\mathrm{CdV}_{2} \mathrm{O}_{4}$ and $\mathrm{MgV}_{2} \mathrm{O}_{4}$ obtained from $M(T, H)$ and $\Delta P(H)$ measurements. Abbreviations PM, PE, and FE are for paramagnetic, paraelectric, and ferroelectric state. The shaded area is the FE state, and the lined area represents a mixed PE-FE state due to the polycrystalline nature of $\mathrm{CdV}_{2} \mathrm{O}_{4}$. The FE phase boundary is inferred from the maximum of $d \Delta P / d \mu_{0} H$. field along the hard axis is not reached by $65 \mathrm{~T}$. The phase diagram includes peaks in $d \Delta P / d \mu_{0} H$ and the onset of the deviation from linear behavior in $M(H)$ (determined by intersecting the linear behaviors below and above the upturn).

We now introduce a model Hamiltonian that includes the effect of the tetragonal and trigonal distortions:

$$
\begin{aligned}
\mathcal{H}= & J \sum_{\langle i j\rangle}^{x y} \mathbf{S}_{i} \cdot \mathbf{S}_{j}+J^{\prime} \sum_{\langle i j\rangle}^{y z, z x} \mathbf{S}_{i} \cdot \mathbf{S}_{j}-D \sum_{i}\left(\mathbf{S}_{i} \cdot \hat{\mathbf{n}}_{i}\right)^{2} \\
& +J_{3} \sum_{\langle\langle\langle i j\rangle\rangle\rangle} \mathbf{S}_{i} \cdot \mathbf{S}_{j}-g \mu_{B} \sum_{i} \mathbf{H} \cdot \mathbf{S}_{i} .
\end{aligned}
$$

$J$ is the NN AFM exchange that couples the $\mathrm{V}^{3+}$ spins along the $x y$ chains, and $J^{\prime}$ is the $\mathrm{NN}$ interaction along $x z$ and $y z . D$ is the single-ion anisotropy, $\hat{\mathbf{n}}_{i}$ is a unit vector along the easy axis, $J_{3}$ is the third NN exchange, $g$ is the gyromagnetic factor, and $\mu_{B}$ is the Bohr magneton.

As in other vanadium spinels [7,9], $J$ and $J^{\prime}$ depend on the occupancy of the relevant $d$ orbitals. The occupancy of the $d_{x y}$ orbital and, thus, the strength of $J$, becomes dominant after the tetragonal distortion below $T_{S} . J^{\prime}$ is determined by the orbital ordering of the remaining $d_{x z}$ and $d_{y z}$ orbitals. Two types of orbital states have been proposed for vanadium spinels: staggered real [7] and a uniform complex orbital ordering [8]. Both cases result in $\left|J^{\prime}\right|<J$. Because $J^{\prime}$ is also geometrically frustrated, the final 3D ordering depends on residual perturbations. Here we consider two competing perturbations: the Zeeman coupling to the external field and a third NN exchange $J_{3}$. The third NN pairs are separated by twice the NN distance on the same $\langle 110\rangle$ chains [Fig. 1(a)].

The $D$ term originates from the relativistic spin-orbit interaction. We estimate the orientation of the easy axes $\hat{\mathbf{n}}_{i}$ and the anisotropy strength $D$ by diagonalizing the singleion Hamiltonian $\mathcal{H}_{t_{2 q}}=\Delta \hat{\lambda}_{8}+\delta\left(\hat{\lambda}_{1}+\hat{\lambda}_{4}+\hat{\lambda}_{6}\right)-\lambda \mathbf{L}^{\prime} \cdot \mathbf{S}$ in the two $d$-electron basis. $\hat{\lambda}_{i}$ are Gell-Mann matrices, $\mathbf{L}^{\prime}$ is the effective angular momentum of length $L^{\prime}=1$ for the $t_{2 g}^{2}$ electronic configuration, and $\lambda \approx 20 \mathrm{meV}$ is the effective spin-orbit coupling constant. The crystal-field splitting estimated from our $a b$ initio calculations $(\Delta \approx 350 \mathrm{meV}$ and $\delta \approx 250 \mathrm{meV}$ ) leads to $D \approx 15.5 \mathrm{meV}$ and an easy axis $\hat{\mathbf{n}}_{i}$, which is tilted about $\theta=35^{\circ}$ from the $z$ axis toward the local $\langle 111\rangle$ direction. Specifically, the easy axes at the four sublattices of the pyrochlore are $\hat{\mathbf{n}}_{m}=\cos \theta \hat{\mathbf{z}}+\sin \theta \hat{\mathbf{e}}_{m}$, where the in-plane unit vectors $\hat{\mathbf{e}}_{m}$ point along the [110], [110], [1 $\left.1 \overline{1} 0\right]$, and [ $\left.\overline{1} \overline{1} 0\right]$ directions for $m=0,1,2,3$, respectively (see Fig. 1).

We first consider the zero field magnetic order. The frustration of the $J^{\prime}$ couplings between the crossing $x y$ chains is relieved by $J_{3}$. For zero trigonal distortion, $\delta=0, J_{3}$ favors a collinear $\uparrow \uparrow \downarrow \downarrow$ ordering of spins along the $y z$ and $x z$ chains (the third neighbor pairs on the $x y$ chains are parallel to each other due to a dominant $J$ ), giving rise to the $\mathbf{Q}=$ $2 \pi(0,0,1) 3 \mathrm{D}$ ordering shown in Fig. 1(a). To characterize 
the AFM order in the pyrochlore lattice, we introduce two Néel order parameters $\mathbf{L}_{x}=\mathbf{S}_{0}+\mathbf{S}_{1}-\mathbf{S}_{2}-\mathbf{S}_{3}$ and $\mathbf{L}_{y}=\mathbf{S}_{0}+\mathbf{S}_{2}-\mathbf{S}_{1}-\mathbf{S}_{3}$ for a tetrahedron [28], where $\mathbf{S}_{m}$ is the magnetization of sublattice $m$. The corresponding values for the structure shown in Fig. 1(a) are $\mathbf{L}_{x}\left(\mathbf{r}_{\mathrm{A}}\right)=$ $4 S \hat{\mathbf{z}} e^{i \mathbf{Q} \cdot \mathbf{r}_{\mathrm{A}}}$ and $\mathbf{L}_{y}\left(\mathbf{r}_{\mathrm{B}}\right)=4 S \hat{\mathbf{z}} e^{i \mathbf{Q} \cdot \mathbf{r}_{\mathrm{B}}}$, where $r_{\mathrm{A}}$ and $r_{\mathrm{B}}$ are coordinates of tetrahedra of types $\mathrm{A}$ and $\mathrm{B}$, respectively. Because $\exp \left(i \mathbf{Q} \cdot \mathbf{r}_{A, B}\right)= \pm 1$, the sign of the Néel order parameters alternate between successive layers.

The trigonal crystal field $\delta$ rotates the easy axis and gives rise to a net magnetization $\mathbf{M}=\mathbf{S}_{0}+\mathbf{S}_{1}+\mathbf{S}_{2}+\mathbf{S}_{3}$ in each tetrahedron. This small FM component is modulated, $\mathbf{M}\left(\mathbf{r}_{\mathrm{A}}\right) \approx 4 S \sin \theta \hat{\mathbf{y}} e^{i \mathbf{Q} \cdot \mathbf{r}_{\mathrm{A}}}, \mathbf{M}\left(\mathbf{r}_{\mathrm{B}}\right) \approx 4 S \sin \theta \hat{\mathbf{x}} e^{i \mathbf{Q} \cdot \mathbf{r}_{\mathrm{B}}}$, and the net magnetization vanishes, $\langle\mathbf{M}(\mathbf{r})\rangle=0$. The tetragonal symmetry is preserved by this magnetic order as the system is invariant under a $\pi / 2$ rotation in the $x y$ plane $(A \leftrightarrow B)$ accompanied by the exchange $\mathbf{L}_{x} \leftrightarrow \mathbf{L}_{y}$. Because the $\mathbf{Q}=$ (001) order is selected by $J_{3}$ out of many degenerate states consisting of decoupled AFM $x y$ chains, the Zeeman coupling to a large enough magnetic field should overcome $J_{3}$ and select the state with finite $\mathbf{M}$ that is shown in Fig. 1(b). We suggest that the transition between these two ordered phases explains our measurements. The magnetic order in Fig. 1(b) has a wave vector $\mathbf{Q}=0$; i.e., all tetrahedra are in the same magnetic structure. This state is described by magnetic order parameters: $\mathbf{L}_{x}(\mathbf{r}) \approx 4 S \cos \theta \hat{\mathbf{z}}, \mathbf{L}_{y}(\mathbf{r})=0$, and $\mathbf{M}(\mathbf{r})=4 S \sin \theta \hat{\mathbf{y}}$, for both types of tetrahedra. The tetragonal symmetry is broken in this case and the Zeeman energy gain is $E_{Z}=4 S g \mu_{B} \sin \theta H$ per tetrahedron for a transverse magnetic field $\mathbf{H}=(0, H, 0)$. A first-order quantum phase transition, thus, takes place at $g \mu_{B} H_{c} \approx$ const $\times J_{3} / \sin \theta$ that removes the polarization by suppressing the $\uparrow \uparrow \downarrow \downarrow$ structure.

We note that the essential physics discussed above is largely independent of the specific values of these parameters. In particular, these parameters are expected to be similar in the two compounds discussed here (e.g., the Néel temperature $T_{N}=42 \mathrm{~K}$ and $33 \mathrm{~K}$ for $\mathrm{Mg}$ and $\mathrm{Cd}$ vanadates, respectively). The critical fields are, thus, of similar order in both cases, as indeed observed experimentally.

We verified the above picture by performing classical Monte Carlo (MC) simulations of $\mathcal{H}$ for $J^{\prime}=0.16 J$, $J_{3}=0.01 \mathrm{~J}$, and $D=0.7 \mathrm{~J}$. $(S=1 \mathrm{spins}$ are approximated by classical unit vectors.) We use the standard Metropolis algorithm and periodic boundary conditions for lattices of up to $N_{s}=16 \times 8^{3}$ spins. Figure 5(a) shows the $M(H)$ curves for $\mathbf{H} \| \hat{\mathbf{y}}$ obtained at different temperatures. A sharp discontinuity at $g \mu_{B} H \approx 0.4 J$ and $T=0$ indicates a firstorder transition. The discontinuity decreases with increasing temperature and disappears at $T \approx 0.15 \mathrm{~J}$. The resulting $H-T$ phase diagram [Fig. 5(b)] includes the two ordered phases shown in Figs. 1(a) and 1(b). The transition to the high- $T$ paramagnetic phase is always continuous (the phase boundaries were estimated by the crossing of the Binder's cumulant). The first-order line between the two

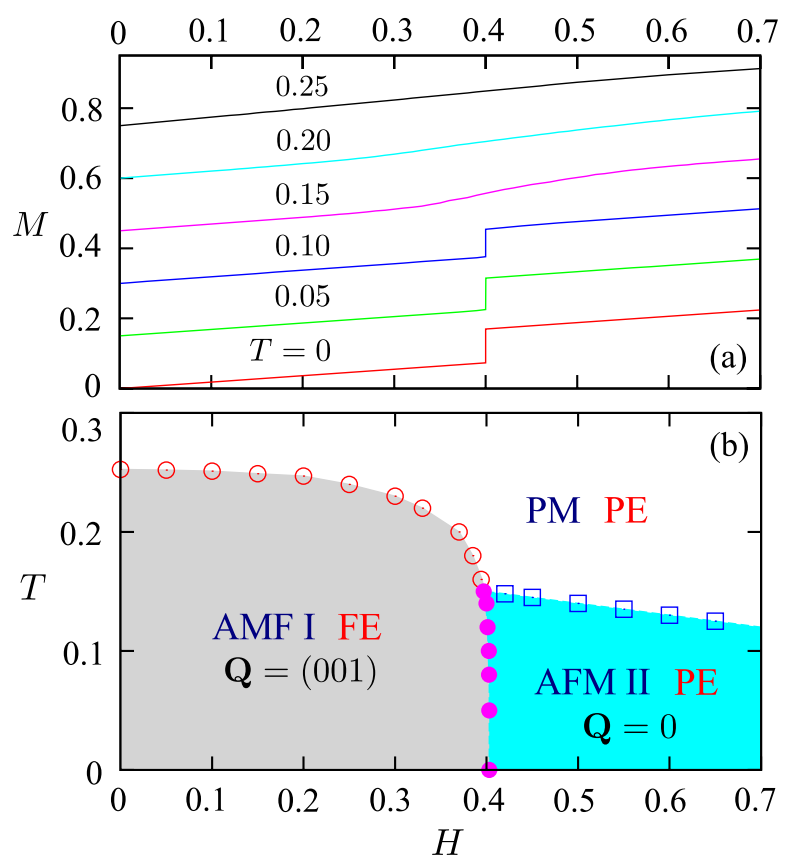

FIG. 5 (color online). (a) $M(H)$ along the $x$ or $y$ directions at various temperatures. The field is measured in units of $J / g \mu_{B}$. (b) $T-H$ phase diagram of model (1) obtained from MC simulations. $T$ is measured in units of $J$. The open circles and squares denote second-order phase boundaries, while filled circles mark a first-order transition line. The three phase boundaries intersect at a bicritical point.

ordered phases was determined with the method of mixed initial state (each of the two coexisting orders occupies half of the lattice) [29]. Relating $T_{c}(H=0)$ with the experimental value of $T_{c} \approx 33 \mathrm{~K}$ (Fig. 4), we estimate a transition field $\mu_{0} H_{c} \approx 47 \mathrm{~T}$, in good agreement with our measurements. The first-order transition is consistent with the magnetic hysteresis observed in $\mathrm{MgV}_{2} \mathrm{O}_{4}$ (see Supplemental Material [26]).

In summary, we observe a field induced phase transition marked by a magnetization jump at $H \approx 40 \mathrm{~T}$ in a single crystal of $\mathrm{MgV}_{2} \mathrm{O}_{4}$ and an upturn in the $M(H)$ slope of polycrystalline $\mathrm{CdV}_{2} \mathrm{O}_{4}$. We also present a model, which includes the effect of orbital degrees of freedom, lattice distortion, and spin-orbit interactions, and predicts a field induced $\mathbf{Q}=0$ magnetic order that is stabilized by relatively small magnetic fields in comparison to the dominant exchange. The measured field induced transition is attributed to the onset of the $\mathbf{Q}=0$ order. Contrary to the $\mathbf{Q}=$ $2 \pi(0,0,1)$ zero field magnetic ground state, the new field induced state does not support ferroelectricity, in agreement with the suppression of $\mathbf{P}$ that we observe in the multiferroic spinel $\mathrm{CdV}_{2} \mathrm{O}_{4}$. This $\mathbf{Q}=0$ state is only possible in the presence of a small trigonal distortion, which has been observed in many vanadium spinels. For example, the trigonal distortion is essential for stabilizing the staggered orbital order of the ferrimagnetic vanadate $\mathrm{MnV}_{2} \mathrm{O}_{4}$ $[21,22]$. Our theory, thus, underscores the importance 
of including the trigonal distortion that exists in both zero and applied fields for describing the magnetism of vanadium spinel compounds. In particular, the presence of the trigonal distortion in vanadium spinels indicates that the orbital ordering in the vanadium spinels family is most likely of the staggered type [7], as in the case of $\mathrm{CdV}_{2} \mathrm{O}_{4}$. Finally, because the $\mathbf{Q}=0$ state also breaks the tetragonal symmetry, a similar phase transition might also be driven by epitaxial strain.

We thank N. Perkins and D. Khomskii for useful discussions and Brian Scott for single-crystal orientations. Work at the LANL was performed under the auspices of the U.S. DOE Grant No. DE-AC52-06NA25396 through the LDRD program. The NHMFL facility is funded by the NSF through cooperative Grant No. DMR-1157490, by the DOE, and by the State of Florida. This material is based upon work supported in part by the NSF under Grant No. PHY-1066293 and the hospitality of the ACP. V. P. acknowledges financial support from the Ramon y Cajal Program. F. R. acknowledges support from Xunta de Galicia 2012-CP071.

[1] R. Moessner and A. P. Ramirez, Phys. Today 59, No. 2, 24 (2006).

[2] Y. Ueda, N. Fujiwara, and H. Yasuoka, J. Phys. Soc. Jpn. 66, 778 (1997).

[3] M. Reehuis, A. Krimmel, N. Bttgen, A. Loidl, and A. Prokofiev, Eur. Phys. J. B 35, 311 (2003).

[4] S. H. Lee, D. Louca, H. Ueda, S. Park, T. J. Sato, M. Isobe, Y. Ueda, S. Rosenkranz, P. Zschack, J. Iniguez, Y. Qiu, and R. Osborn, Phys. Rev. Lett. 93, 15640 (2004).

[5] E. M. Wheeler, B. Lake, A. T. M. Nazmul Islam, M. Reehuis, P. Steffens, T. Guidi, and A. H. Hill, Phys. Rev. B 82, 140406 (2010).

[6] A. N. Vasiliev, M. M. Markina, M. Isobe, and Y. Ueda, J. Magn. Magn. Mater. 300, e375 (2006).

[7] H. Tsunetsugu and Y. Motome, Phys. Rev. B 68, 060405 (2003); Y. Motome and H. Tsunetsugu, ibid. 70, 184427 (2004).

[8] O. Tchernyshyov, Phys. Rev. Lett. 93, 157206 (2004).

[9] S. Di Matteo, G. Jackeli, and N. B. Perkins, Phys. Rev. B 72, 020408 (2005).

[10] T. Maitra and R. Valenti, Phys. Rev. Lett. 99, 126401 (2007).

[11] G. Giovannetti, A. Stroppa, S. Picozzi, D. Baldomir, V. Pardo, S. Blanco-Canosa, F. Rivadulla, S. Jodlauk,
D. Niermann, J. Rohrkamp, T. Lorenz, S. Streltsov, D. I. Khomskii, and J. Hemberger, Phys. Rev. B 83, 060402(R) 2011).

[12] Ch. Kuntscher, K. Rabia, M. K. Forthaus, M. M. Abd-Elmeguid, F. Rivadulla, Y. Kato, and C. D. Batista, Phys. Rev. B 86, 020405(R) (2012).

[13] V. Pardo, S. Blanco-Canosa, F. Rivadulla, D. I. Khomskii, D. Baldomir, H. Wu, and J. Rivas, Phys. Rev. Lett. 101, 256403 (2008).

[14] Y. Kato, G.-W. Chern, K. A. Al-Hassanieh, N. B. Perkins, and C.D. Batista, Phys. Rev. Lett.108, 247215 (2012).

[15] G.-W. Chern and C. D. Batista, Phys. Rev. Lett. 107, 186403 (2011).

[16] A. P. Ramirez, B. S. Shastry, A. Hayashi, J. J. Krajewski, D. A. Huse, and R. J. Cava, Phys. Rev. Lett. 89, 067202 (2002).

[17] R. Higashinaka and Y. Maeno, Phys. Rev. Lett. 95, 237208 (2005).

[18] Y. Tabata, H. Kadowaki, K. Matsuhira, Z. Hiroi, N. Aso, E. Ressouche, and B. Fak, Phys. Rev. Lett. 97, 257205 (2006).

[19] K. C. Rule, J. P. C. Ruff, B. D. Gaulin, S. R. Dunsiger, J. S. Gardner, J. P. Clancy, M. J. Lewis, H. A. Dabkowska, I. Mirebeau, P. Manuel, Y. Qiu, and J. R. D. Copley, Phys. Rev. Lett. 96, 177201 (2006).

[20] L. Yin, J. S. Xia, Y. Takano, N. S. Sullivan, Q. J. Li, and X. F. Sun, Phys. Rev. Lett. 110, 137201 (2013).

[21] S. Sarkar, T. Maitra, R. Valenti, and T. Saha-Dasgupta, Phys. Rev. Lett. 102, 216405 (2009).

[22] G.-W. Chern, N. Perkins, and Z. Hao, Phys. Rev. B 81, 125127 (2010).

[23] S. Sarkar and T. Saha-Dasgupta, Phys. Rev. B 84, 235112 (2011).

[24] A. N. Yaresko, Phys. Rev. B 77, 115106 (2008).

[25] M. Onoda and J. Hasegawa, J. Phys. Condens. Matter 15, L95 (2003).

[26] See Supplemental Material at http://link.aps.org/ supplemental/10.1103/PhysRevLett.112.017207 for the difference in the critical field between first and subsequent magnetic field pulses.

[27] A. T. M. Nazmul Islam, E. M. Wheeler, M. Reehuis, K. Siemensmeyer, M. Tovar, B. Klemke, K. Kiefer, A. H. Hill, and B. Lake, Phys. Rev. B 85, 024203 (2012).

[28] G.-W. Chern, C. J. Fennie, and O. Tchernyshyov, Phys. Rev. B 74, 060405(R)(2006).

[29] M. Creutz, L. Jacobs, and C. Rebbi, Phys. Rev. D 20, 1915 (1979). 